\title{
Biological Chemistry in Intermediate Lipid Metabolism Disorder
}

\author{
ALINA PLESEA CONDRATOVICI ${ }^{1}$, ALINA MIHAELA ELISEI ${ }^{1}$, DECEBAL VASINCU ${ }^{2 *}$, IULIAN DAN CUCIUREANU², AUREL NECHITA ${ }^{1 *}$, \\ CAMELIA ANA GRIGORE ${ }^{1}$, CATALIN PLESEA CONDRATOVICI ${ }^{1}$ \\ 'Dunarea de J os University of Galati, Medicine and Pharmacy Faculty, 47 Domneasca Str., 800008, Galati, Romania \\ ${ }^{2}$ Grigore T. Popa University of Medicine and Pharmacy, 16 Universitatii Str.,700115, Iasi, Romania
}

\begin{abstract}
Any pathological process is accompanied by quantitative and qualitative changes in metabolism, which is the main form of life manifestation. Metabolism disorders (it is the permanent exchange of substances between the body and the environment) arise if the activity of the central nervous system is affected; the trophic function of the nervous system directs nutrition and metabolism. In this function, the coordinating role belongs to the central nervous system and is made by means of the endocrine glands. Lipids introduced into the body are digested mainly with the help of the pancreatic and intestinal juice and are resorbed through the walls of the small intestine. Even in the intestinal wall, the re-synthesis of fatty acids and glycerine fat occurs. A certain amount of neutral fat is probably resorbed without being split into fatty acids and glycerine. Fats are mainly resorbed through the lymphatic system, in part (about 30\%) by means of the portal vein system; the entire fat emulsion penetrates into the blood and its main mass is deposited in certain fatdeposits: the adipose subcutaneous cell tissue, the epiploon and the mesenterium of the abdominal cavity, as well as in the fatty layers of the various organs. In fat deposits, processes of lipid formation from carbohydrates and of transformation of higher fatty acids can occur. Lipids from fat deposits are subject to oxidation, especially at the liver level.
\end{abstract}

Keywords: metabolic disorders, nervous system, fats re-synthesis, obesity, cholesterol, lipids, stroke

The regulation of lipid metabolism is achieved through the nervous system. A proof of this is the blocking of lipid mobilization from fat deposits, the acceleration of the disappearance of fats accumulated in the liver due to the decrease in the amount of glycogen from the liver after liver denervation or after cutting of the spinal cord above the sixth cervical segment. The central area of lipid metabolism regulation would be in the hypothalamic region. Ablation of cerebral hemispheres in pups causes changes in the lipid content in the organs.

In pigeons, after anterior brain ablation, fat storage and body weight are increased. Among the endocrine glands, especially the anterior lobe of pituitary gland, pancreas, adrenal glands and gonads are those that influence lipid metabolism and fat deposits, as endocrine glands represent intermediarylinks in nerve regulation of lipid metabolism.

Lipidosis is a disorder of lipid metabolism, where enzymatic defects and of other proteins underlie lipid accumulation (and storage).

\section{Resorption and fat depositing disorders}

Lipid metabolism disorders include lipidosis and those diseases characterized by serum lipoprotein concentration and blood lipid transport abnormalities. The lipids are transported into the blood in the form of globular molecular complexes called lipoproteins. Their surface consists of amphipathic lipids (phospholipids and non-esterified cholesterol); triglycerides and cholesterol esters, the form under which cholesterol is transported and stored.

Lipid digestion and resorption may be disturbed by various causes: in the course of the disorder of external pancreas secretion due to insufficient secretion or complete lack of bile, due to an impaired motor function of the intestines (accentuated peristalsis), or due to a decreased capacity of the epithelium to actively resolve lipids. The presence in the faeces of $20 \%$ of the food fats (instead of $5-10 \%$, normally) is considered as pathological.

Chylomicrons transport lipids from the intestine (by means of intestinal lymphatic pathways) to the periphery (skeletal muscles, adipose tissue) where it activates the endothelial lipoprotein-lipase; there is a dissociation between free fatty acids that are taken over by myocytes and adipocytes.

In the liver, chylomicron residues bind to receptors (the protein associated with LDL receptors), are enriched by endocytosis supplying TG, cholesterol and cholesterol esters. TG and cholesterol as well as those synthesized by novo are exported by the liver in the form of very low-density lipoproteins towards the periphery, where lipoprotein-lipase finds itself, which causes the release of fatty acids. At the liver level, they are again loaded with lipids and leave the liver in the form of very low-density lipoproteins.

The depositing, retention and occurrence of fatin cells is found during various pathological processes. Deposition of fats in cells is possible due to infiltration with fat brought by means of lymph and blood, or due to fatty degenerationthe association of fat infiltration with protoplasm destruction. It is also possible to have a fatty decomposition: the occurrence of fat due to the disorder of the physicochemical state of the protoplasm, which conditions the release of lipids from their binding to the proteins. The causes of fatty infiltration and dystrophy can be the following: the action of various toxic agents (phosphorus and arsenic), of toxins, insufficient oxygen intake towards tissues (hypoxia), starvation; fat loss in tissues disorder. In the first case there is a decrease in the oxidative processes in tissues that prevent oxidation of fats or a disorder in the splitting of neutral fats. In the second case, the pathogenesis of fatty infiltration (of the liver) is related to the lack of choline, methionine and of other socalled lipotropic substances in the diet.

*email: decebal.vasincu@umfiasi.ro; aurelnechita@yahoo.com 
The mechanism of lipotropic action of choline consists in that this substance takes part in the synthesis of phospholipids and thereby stimulates the output of fat from the liver. Methionine participates in the formation of choline by the release of its methyl group. The occurrence of the fatty liver infiltration can be prevented by administering, along with food, lecithin (which contains choline) and methionine (which is abundant in casein).

Lipemia - The amount of fat and lipoids in the blood varies between 0.2 and $0.8 \%$ in humans. Insufficient or excess lipid administration in the body as well as their resorption disorder influences their blood concentration. Lipemia may also vary as a result of disturbance of intermediate lipid metabolism.

The increase of fat concentration in the blood, often parallel to the increase in the concentration of lipoid substances, is called lipemia, and the increase in the amount of lipoids, lipoidemia.

One of the causes that generates lipemia or lipoidemia is the increased food intake of lipids and lipoids; the constant lipemia in these cases (food or provoked lipemia) reaches the maximum within 6 hours after food intake and disappears within 9-10 hours. The pathological processes that cause lipemia include: starvation, diabetes, haemorrhage, alcoholism, chloroform, ether intoxication, some toxic gases, and various liver diseases. Lipoidemia is found in all of these lipemias. Intoxication with alcohol, chloroform, ether causes the increase in lipoids in the blood (cholesterol and phosphatides). At the base of the lipemia pathogenesis, there is often a disorder of the transport of fat from the fat deposits in the organs and vice versa (transportlipemia), as well as the delayed passage of blood lipids into the organs or the retention of lipids in the blood (retentive lipemia) (in anemia) [1-4].

Lipids can appear in the urine in sufficient quantities in order to be observed: lipuria. This happens in the so-called lipoid nephrosis, accompanied by lipemia and cholesterolemia. It is very pronounced in cases where the chyliferous vessels join together with the urinating tubes, and that is why this phenomenon is called chyluria. This is found in certain tropical diseases, pancreatic tumours and inflammatory processes in the abdominal cavity.

\section{The intermediate lipid metabolism disorder}

Intermediate metabolism represents the total transformation that foods introduced into the body (carbohydrates, proteins, water, minerals) suffer. Carbohydrates are absorbed from the intestine in the form of monosaccharides, the main product being glucose, which gets through the portal circulation to the liver, which converts part of the glucose into (deposit) glycogen, a process called gluconeogenesis. The storage of glycogen is done in the liver and in the muscles. Glycogen may also be synthesized from non-glucose sources (proteins) and the process is called gluconeogenesis. In this process the liver has the main role. The catabolism of carbohydrates is carried out in glycogenolysis processes, the depolymerization of glycogen by gradual detachment of glucose molecules. Glucose is found in the blood at a concentration of $70-100 \mathrm{mg} \%=$ glycemia. If higher than 130 - 140 diabetes may occur. Carbohydrate degradation is done in two phases: anaerobic, aerobic.

The firstanaerobic phase is anaerobic glycolysis in which glucose is phosphorylated up to pyruvic acid from which it then turns to lactic acid. Anaerobic degradation of carbohydrates up to lactic acid allows the body to perform a work in relative anoxia conditions as it happens in case of short efforts. The lactic acid formed in the muscles in very short efforts follows three ways: - if the muscle does not receive 02 the lactic acid accumulates and causes acidosis that inactivates the enzymes disturbing the muscle contraction; in the presence of $02-75 \%$ of the formed lactic acid is oxidized in the muscle cell the pyruvic acid getting restored; $10 \%$ of the lactic acid passes into the blood and takes part in the synthesis of glycogen.

In the second anaerobic phase, glycolysis takes place in the cytoplasm and forms an A.T.P. (energy) molecule, namely $56 \mathrm{Kcal} / \mathrm{mol}$ of glucose [6].

The aerobic phase starts from two molecules of pyruvic acid that convert to acetyl coenzyme $A$, and the degradation continues in the Krebs cycle within decarboxylation and dehydrogenation, $\mathrm{CO} 2$ is released that will be eliminated by breath. $\mathrm{H}$ atoms are activated in the respiratory chain and will react with 02 forming $\mathrm{H} 2 \mathrm{O}$. In the aerobic phase degradation is complete in $\mathrm{CO} 2, \mathrm{H} 2 \mathrm{O}$ and energy. Stored in 38mol A.T.P. which represents 688 $\mathrm{Kcal} / \mathrm{mol}$ of glucose.

Water Metabolism - After 02, water is the second essential ingredient in maintaining life. The need for water $-2.5 \mathrm{~L}$ / day depending on the temperature and the effort made and can reach $4 \mathrm{~L}$ in $24 \mathrm{~h}$.

The need for mineral substances is on average of 25 grams of which salt represents 10 grams. Na.- is found in the highest amount in the blood, in the interstitial fluid, and the remaining of $5-10 \%$ in the cells and $25 \%$ in the bone. The importance of $\mathrm{Na}$ in the body resides in achieving osmotic blood pressure in processes of cellular excitability, in nervous conductivity, in membrane permeability, and in the performance of the buffer system in order to maintain the $\mathrm{pH}$ of the intern environment. Potassium - can be found in high quantity in the cell and $\mathrm{Na}$ in the intercellular space and helps maintain neuro-muscular excitability. Ca can be found in muscles, blood - is brought along with food, and its intestinal absorption is made in the presence of vitamin D. Phosphorus - is predominant in the chemical composition of the bones, is absorbed in food. It has a role in the formation of the skeleton, the membrane structure, having a strong energetic role $[7,8]$.

One of the manifestations of the intermediate lipid metabolism disorder is the elimination of ketone bodies through urine (acetoacetic acid, â-oxybutyric acid and acetone), so-called acetonuria, as well as the increase in ketone bodies of the blood content, the so-called acetonemia.

The source of formation of ketone bodies, both in the normal state and in pathological processes, is represented by fatty acids. Due to deamination and oxidation certain amino acids can be a source of ketone bodies formation.

Ketone bodies are generally formed in the liver. The exhaustion of glycogen reserves in the liver is accompanied bylipid accumulation in this organ and formation of ketone bodies, in case of qualitative starvation or diabetes. Acetonemia can occur in relation to unilateral nourishment through lipids.

Accumulation of ketone bodies in the blood and tissues causes the occurrence of acidosis.

Generalized obesity-in the body we can find regional and generalized pathological fat deposits, the weight of the body rising and being able to reach up to $180 \mathrm{~kg}$ and more.

The sources of lipids in the body are food fats and synthesized fats from intermediate products of carbohydrate metabolism.

The pathogenetic factors in obesity may be the following: 
Excessive appetite. It is related to the disorder of central nervous system regulation activity, with the appearance of a stinging excitation outbreak in the food centre. The inconsistency between the amount of food ingested and the feeling of satiety leads in these cases to the fact that the energy intake proves to be greater than its consumption, due to which fact the accumulation of fat in the body occurs.

Muscle activity disorder. Adynamia, the limitation of muscle movements, contributes to lower energy spending. After insufficient muscle activity, energy intake exceeds its spending and fat depositing occurs. The decrease in muscle activity can also be a consequence of obesity. In this case, obesity becomes the factor that contributes to the accumulation of fats due to the limitations of the movements encountered in the obese.

Tissue lipophilia ( tissue affinity to fat) that is, the property of fat deposits to accumulate lipids. In these cases, even in the case of increased basal and energetic metabolism, the body mainly consumes proteins and carbohydrates, and lipids to a lesser extent, due to the inhibition of lipid mobilization. Solid lipid fixation in a fat deposit may be a result of the nervous regulation disorder of fat mobilization processes. Lipophilia also often explains regional lipid deposits. In this way, in the accumulation of fats, the peripheral tissues in which these deposits are produced play an important role.

The occurrence of any form of obesity is usually associated with several factors. In case of association of increased appetite with insufficient muscle activity, the development of obesity is observed even if each of these factors mentioned above does not far exceed the normal limits.

The disturbance of the oxidative capacity of tissues, which is determined by basal metabolism research and by studying the specific dynamic action of foods.

We have to bear in mind that the existing methods for basal metabolism determination do not always offer us a fair picture of the real intensity of the oxidative processes; even in the case of an equal surface area of the body and in the case of equal body weight, the basal metabolism of different individuals may vary according to individual peculiarities (nervous system condition, way of life, character of activity, etc.). Besides, in many forms of obesity one can observe the retention of water in the body. This often leads to mistakes in the appreciation of the real role of changes in basal metabolism in the production of obesity. On the basis of modern research we must recognize that the decrease of metabolism in the body is not the main pathogenic factor of all forms of obesity.

The influence of the modification of the specific dynamic action of foods on the occurrence of obesity cannot be fully established, and in cases where the specific dynamic action of the diet is low, favourable conditions for the accumulation of fats may occur.

The etiology of obesity is different. Among the various causes of obesity, we will name the disorder of central nervous system and endocrine glands regulation activity, which in turn are regulated by the central nervous system.

The change of the cortical regulation of the oxidative processes in the body is of great importance in the occurrence of obesity. This is confirmed by clinical observations on cases of obesity arising due to certain neuropsychiatric conditions.

There are also other forms of obesity of centrogenic origin; disorders of diencephalon function in tumours or trauma in the grey nucleus region in the vicinity of pituitary gland can cause the development of fatty deposits. Asymmetric and regional fat deposits occur most often due to nervous system disorder, especially to its sympathetic segment. Experimentally, animal obesity occurrence could be produced after damage to the hypothalamic region. Among the obesities of endocrine origin are the pituitary, hypogenital, thyroid and insular forms.

Pituitary obesityis characterized by the depositing of fat in certain places, especially in the region of the pelvis, of the abdomen, in the pubic region, the upper portion of the thighs and shoulders; there are no fat deposits in the extremities' flexion places. The cause of this form of obesity is the change of the anterior pituitary lobe function. The pathogenicity of fat depositing in case of pituitary obesity is not yet well studied.

Hypogenital obesity occurs as a result of genital gland hypofunction, a fact known from the observations made on the castrated. This form of obesity is characterized by certain features: fat is mainly disposed in the region of buttocks, abdomen, in the lumbar, thoracic region, and on the lower area of thighs. The poor development of secondary sexual signs draws attention. The pathogenesis of this form of obesity consists of a general decrease in the oxidative processes in the body, the basal metabolism being usually at the lower limit of normal variations. Physiologically, a certain accumulation of fat is sometimes found in women during the climacteric period, when the function of the sexual glands is weakened.

Thyroid gland disorder (its hypofunction) influences the intensity of the oxidative processes in the body. Decreased thyroid function accompanied by decreased basal metabolism predisposes to obesity. This is confirmed by cases where thyroid gland hypofunction is associated with obesity; certain forms of obesity are rudimentary forms of hypothyroidism, which in more obvious cases lead to the development of myxedema.

Enhanced function of the insular apparatus of the pancreas can cause generalized obesity. Insulin hypersecretion leads to increased glycogen synthesis and to increased metabolism of carbohydrates into lipids, being accompanied by a constant sensation of hunger, which contributes to increased food intake. One of the signs of increased insulin secretion is a less marked increase in the glycemic gradient after glucose administration. The degree of decrease of the glycemic gradient after its growth is an index of the amount of hormones that penetrate into the blood in response to pancreas excitement through glucose intake. The hypoglycaemic phase of the glucose gradient is explained by the action of insulin elaborated in response to glucose intake, and which is always highlighted after glucose intake. In hyperinsulinemia, this phase is particularly marked.

The emergence of endocrine forms of obesity should not represent a result of the gland.

In the emergence of obesity, other factors also have an important role: the ingestion of a large amount of food, as well as all the causes that cause the lack of sufficient movement. Chronic intoxications also represent causes of obesity, as a result of which the tissue oxidative processes are diminished, and energy intake is increased.

\section{Lipid metabolism pathology}

The similarity in terms of solubility as well as the frequent common localization of lipoids and fats, both in normal and pathological conditions, allow us to establish a link between lipoid and lipid metabolism disorders, although lipoids differ from lipids in what regards the chemical and physiological properties. 
In the lipid group, lecithins and cholesterol are included. The lecithin resorption occurs after their prior splitting.

The lecithins' re-synthesis takes place in the walls of the intestine. In the body tissues enzyme splitting processes of lecithins occur, which are carried out in parallel with their synthesis processes, starting from the main chemical components, choline, fatty acids, and glycerophosphoric acid.

Lecithinemia, namelyincreased lecithin content in blood, is often found along with lipemia. The modification of lecithin content of blood is found to be in connection with pathological disorders of general lipid metabolism, related to hematopoietic function disorder and to a number of other functions disorder.

Cholesterol metabolism and its disorders: cholesterol resorption, its transformations into the body, and ways to remove this substance.

In the body, by oxidation, part of the cholesterol turns into colic acids, which are eliminated by the bile; another part is eliminated unchanged. The synthesis of the cholesterol is performed in the human body.

Before being resorbed in the small intestine cholesterol esters, fatty acid cholesterol compounds, split into fatty acids and cholesterol due to the enzymatic action of digestive juices; some of the cholesterol esters can be absorbed directly through the intestinal walls. Cholesterol esters are found in the tissues and body humours in an amount two-fold higher than free cholesterol.

Resorption of cholesterol and its esters occurs only in the presence of lipids.

The occurrence of hypercholesterolemia is related to neuro-endocrine regulation disorders. In dogs, ablation of both cerebral hemispheres causes the lowering of cholesterol in the blood.

Hypercholesterolaemia in humans is seen not only after the intake of certain foods containing large amounts of cholesterol but also during many pathological processes: diabetes mellitus, liver disease, starvation, severe tuberculosis, hypertensive disease, feverish conditions.

The basis of these cholesterolemia is either the disorder of the transport and the elimination of the substance in the body, or the tissue destruction, or the chemical and synthesis cholesterol transformations disorder.

Cholesterol esterification is predominantly performed in the liver. Certain disorders of the liver function are accompanied by a decrease in the blood between the bound cholesterol and the free cholesterol. Some pathological processes occur in connection with cholesterol deposited in the body [9].

Atherosclerosis is linked to the cholesterol metabolism disorder. This condition consists in the thickening of the inner layer of the vascular walls, especially in the large arteries, and in the depositing in this layer of lipoid substances (cholesterol and its esters) [10].

Particular importance is attached to the change in the colloidal state of plasma cholesterol, which is related to the modification of its protein fractions.

In the pathogenesis of atherosclerosis, the modification of the vascular wall is important, a fact due to poisoning or haemodynamic disorders [9-12].

\section{Biological Chemistry}

The links of chemistry with life sciences (biology, medicine) are old and well-known. As early as 1871, in the famous Dictionariulu Limbei Romane by A.T. Laurian and J.C. Massimu, a symbolic work of our Academy, they stated (vol. I, p. 660) that: ... since chemistry became a science, man has come to know more thoroughly the nature of the elements of his body. This interpenetration of chemistry, in particular with biology and medicine, has continued, with periods of impetus and stagnation characteristic to the history of science, up to the present day. In 1907, Nobel laureate Emil Fischer wrote: In its first youth, organic chemistry was closely linked to biology. I consider it not only possible but desirable that this close connection of chemistry with biology be restored so that the great chemical secrets of life are revealed through cooperative work. And therefore, the chemistry/ biology link remained as tight as it used to be (maybe even more) nowadays, nearly 100 years from $E$. Fischer.

Lipids are also referred to as fatty matters or fatty substances. Lipids representan important family of organic substances being present in the structure of any living organism; they are present in higher quantity in the plant reign than in the animal one; they are found in the protoplasm of cells in the form of drops or other inclusions. Some of the lipids form complex unstable combinations with proteins, called lipoproteins. Due to their universal spread, the physiological importance of lipids that are complex organic substances can be deduced. From a chemical point of view, the lipids are esters of certain polyalcohols with organic or mineral acids.

$$
\mathrm{R}-\mathrm{OH}+\mathrm{HOOC}-\mathrm{R}^{\prime}=\mathrm{ROOC}-\mathrm{R}^{\prime}+\mathrm{H}_{2} \mathrm{O}
$$

Some more complex lipids also contain an aminoalcohol in their structure. Lipids are insoluble in water and soluble in nonpolar organic solvents. They are extracted from cells and tissues using organic solvents. Their role in the body is: plastic role-component of the cell membrane, ensuring the transport of substances and cell permeability (phospholipids and cholesterol); the energetic role - the high caloric reserve substance, accumulates in the skin tissue, being a thermal and mechanical insulator; favours the absorption of vitamins $(A, D, K)$ and facilitates the assimilation of proteins. They are indispensable for grow th (essential fatty acids) /

Lipids are classified into two groups: simple, ternary lipids, composed of C, H, O; complex lipids, composed of $\mathrm{C}, \mathrm{H}, \mathrm{O}, \mathrm{P}$ or $\mathrm{P}$ and $\mathrm{N}$. Simple lipids are represented by: glyceride-steroid-cerides-aromatic esters-etholides. Complex lipids: - phospholipids - phosphoaminolipids and include esters, alcohols and hydrocarbons of natural origin insoluble in water. From the point of view of the chemical composition, the lipids are divided into: phospholipid triglycerides.

Chemistry is based on the biological world through an axiom of existence: the mere fact that biological systems and in particular human beings exist, demonstrates the fantastic complexity of the structure and functioning of the molecular world. It shows that such complexity exists, despite our present inability to understand how it operates and how it has appeared (J.-M. Lehn).

\section{Conclusions}

After absorption of substances at the small intestine level these reach the blood ( $80 \%$ lymphatically, the rest through the blood), from where they can be stored in the adipose tissue in the form of reserve lipids, temporarily deposited in the liver or subjected to beta oxidation processes in the tissues.

Hyperlipidaemia is an excess of A.G.L over $15 \mathrm{mg} / \mathrm{dL}$, 0.4-0.6 mEq / L, which occurs by intense lipolysis from: stress, chronic alcoholism, cold, smoking by activating lipolysis, in hyperthyroidism by excess thyroxine; the lipolytic action of catecholamines is enhanced by the 
activation of adenyl cyclase on one side, while phosphodiesterase is inhibited on the other side.

The close correlation between the endogenous and exogenous cholesterol sources, if exogenous cholesterol intake increases, hepatic synthesis is inhibited by HMG$\mathrm{Co} A$ reductase; cholesterol levels depend on: intake; endogenous synthesis; metabolization and consumption. The liver has a major role in degradation and elimination. Stroke and coronary diseases are the most important pathology.

\section{References}

1.BETTERIDGE DJ. High Density Lipoprotein and Coronary Heart Disease. In BMJ 298(6679): 974-5. 1989. Ref Type: J ournal (Full).

2.CLEEMAN JI. Executive Summary of the Third Report of the National Cholesterol Education Program (NCEP) Expert Panel on Detection, Evalution and Treatment of High Blood Cholesterol in Adults. In J AMA 285: 2486-97. 2001. Ref Type: J ournal (Full).

3.ELENA POPA CRISTEA. Metabolismul lipidelor. In Biochimie Medicala. Veronica Dinu, Eugen Trutia, Elena Popa-Cristea, Aurora Popescu, Editura Medicala, Romania, 2 ed., 1998, 394-478.

4.BURLUI, A., GRAUR, M., CONSTANTINESCU, D., CARDONEANU, A., MACOVEI, L., REZUS, E., Rev.Chim.(Bucharest), 69, no.5, p.1279-1282. 5.FRANCES FISCHBACH. Chemistry Studies. In A Manual of Laboratory and Diagnostic Tests. Lippincott Williams \& W ilkins, USA, 8 ed., 2009, 450-452.
6.FLORESCU, L, TEMNEANU, OR, MINDRU, DE. Social and Medical Implications of Teenage Motherhood. REVISTA DE CERCETARE SI INTERVENTIE SOCIALA, 52, 2016, pp. 80-91

7.MINDRU, DE, STANESCU, RS, MATEI, MC, DUCEAC, LD, RUGINA, A, TEMNEANU, OR, UNGUREANU, M, FLORESCU L. Stress in pediatric patients - the effect of prolonged hospitalization. MEDICAL-SURGICAL J OURNAL-REVISTA MEDICO-CHIRURGICALA, 120, 2016, no.2, pp. 417423.

8.MINDRU, DE, MATEI, MC, RUGINA, A, CIOMAGA, IM,NISTOR, N, FLORESCU, L. The informed consent in pediatrics - a child's right. MEDICAL-SURGICAL J OURNAL-REVISTA MEDICO-CHIRURGICALA, 123, 2019, no.1, pp.153-160.

9.FRANCES FISCHBACH. Effects of Drugs on Laboratory Tests. In A Manual of Laboratory and Diagnostic Tests. Lippincott Williams \& Wilkins, USA, 7 ed., 2004, 1243.

10.PALADE, D.O., COBZEANU, B.M., ZAHARIA, P., DABIJA, M., 3D Reconstruction Role in Surgical Treatment of Sinonasal Tumours. Rev Chim (Bucharest), 69, no.6, 2018, pp. 1455-1457

11.GORDON DJ, RIFKIND BM. High Density Lipoprotein-the Clinical Implications of recent studies. In N Engl J Med 321(19): 1311-6. 1989. Ref Type: J ournal (Full).

12.***Laboratory Corporation of America. Directory of Services and Interpretive Guide. High-Density Lipoprotein Cholesterol. www.labcorp.com 2010. Ref Type: Internet Communication.

13.PETER P TOTH. The Good Cholesterol, High-density Lipoprotein. In Circulation 111: 89-91. 2005. Ref Type: Journal (Full).

$\overline{\text { Manuscript received:29.12.2018 }}$ 